\title{
煤矿地面物探工程测量中 GNSS RTK 操作方法及步骤
}

\section{Operation Method and Steps of GNSS RTK in Engineering Survey of Coal Mine Surface}

\section{Geophysical Exploration}

\section{何云 ${ }^{1}$ 何金能 ${ }^{2}$}

Yun $\mathrm{He}^{1}$ Jinneng $\mathrm{He}^{2}$

\section{1.云南弥勒吉成矿业有限公司 \\ 中国·云南 弥勒 652300 ; \\ 2.云南方圆中正工贸有限公司 \\ 中国·云南 昆明 650208 \\ 1. Yunnan Maitreya Jicheng Mining Co., Ltd., \\ Maitreya, Yunnan, 652300, China; \\ 2. Yunnan Fangyuan Zhongzheng Industry and \\ Trade Co., Ltd., \\ Kunming, Yunnan, 650208, China}

【摘 要】主要介绍 GNSS RTK 在地面物探工程测量中的应用, 优化了操作方法及步骤, 精 炼了基准站及移动站校正、放样测量及软件操作等操作程序。使之更好地应用于地面物探 工程测量中

【Abstract】This paper mainly introduces the application of GNSS RTK in the ground geophysical engineering survey, optimizes the operation method and steps, and refines the operation procedures of calibration, setting out survey and software operation of reference station and mobile station. So that it can be better used in ground geophysical survey.

【关键词】工程测量; GNSSRTK; 操作方法及步骤

【Keywords】engineering survey; GNSS RTK; operation method and steps 【DOI】10.36012/se.v2i1.1160

\section{1 引言}

随着 GNSS 技术的快速发展, RTK 测量技术也日益成熟, RTK 测量技术逐步在测绘中得到广泛应用。RTK 测量技术是 个全天性、全天候、全天时、高精度的导航定位和定时传递系 统, 具有准确度高、运算快、效率高、机动灵活、操作简便快捷、 受地形影响小等特点。

\section{2 工程概况及测量设备的选用}

在弥勒市北部矿区煤矿地面物探工作区面积 $4.8 \mathrm{~km}^{2}$, 测 点 1614 个, 地形切割较深。工作区面积大, 测点多, 加之测量 工作主要为放样、测点采集, 选用传统的经纬仪或全站仪测 量, 最少需要两个人合作, 且要求测站点与放样点通视, 若不 通视还需要进行转站。本工程测量选用 G990(RTK) 高精度 GNSS 接收机, 主要配套设备有三脚架、天线、MG758 手簿和 软件等。

\section{RTK 操作及步骤}

\section{1 基准站操作}

基准站的架设有两种方法, 一种是架设在已知点, 另一种 是任意架站。本次地面物探测量工程均采用任意架设基站,选 择视野开阔能覆盖本地面物探区块的位置, 视场内障碍物的 高度角不宜超过 $15^{\circ}$, 远离大功率无线电发射源 (电视台、电 台、微波站等) $200 \mathrm{~m}$ 以上, 距高压输电线和微波无线电信号传
输通道 $50 \mathrm{~m}$ 以上, 附近无强烈反射卫星信号的物件(如大型 建筑物等) ${ }^{[1]}$ 。

基站选址完成, 组装基站, 三脚架整平架设稳固牢靠后, 开始操作基站：打开主机电源 $\rightarrow$ 模式 $\rightarrow$ 基站 $\longrightarrow$ 按电源键确认 选择; 数据链 $\rightarrow$ 外接数据链 $\rightarrow$ 按电源键确认选择; 差分 $\rightarrow$ 差分 格式(RTCM3) 一按电源键确认选择; 启动基准站(自动和手 动两种) $\rightarrow$ 按 F1 功能键进人设置菜单, 选择“开始” (手动) $\rightarrow$ 启动基准站(单点启动: 当前 GPS 坐标为基准站坐标; 上次坐 标: 已保存的基准站坐标 $) \rightarrow$ 保存基准站坐标(实现重复设站) $\rightarrow$ 按电源键确认选择。

\section{2 移动站操作}

当基准站架设成功, 开始移动站连接与设置: 打开主机电 源 $\rightarrow$ 模式 $\rightarrow$ 移动站 $\rightarrow$ 按电源键确认选择; 数据链 $\rightarrow$ 内置电 台 $\rightarrow$ 更改通道 (CHANNEL5 与基准站一致) $\rightarrow$ 按电源键确认 选择; 差分 $\rightarrow$ 差分格式 (RTCM3 与基准站一致) $\rightarrow$ 按电源键确 认选择; 启动移动站 $\rightarrow$ 选择 “开始” $\rightarrow$ 确认 ${ }^{[2]}$ 。

\subsection{MG758 手簿及 eSurvey 软件操作}

基准站和移动站正常工作后, 开始 MG758 手簿及 eSurvey 软件操作: 打开手簿电源 $\rightarrow$ 运行 eSurvey 软件 $\rightarrow$ 新建 (或打开已有文件) $\rightarrow$ 确定 $\rightarrow$ 修改中央子午线参数为当地子午 线数值 $\rightarrow$ 确定 $\rightarrow$ 确定 $\rightarrow$ 关闭; 作业 $\rightarrow$ 设置 $\rightarrow$ 仪器设置 $\rightarrow$ 通信 设置 $\rightarrow$ 蓝牙 (选择配对机号) $\rightarrow$ 连接 $\rightarrow$ 关闭(如没有匹配机号: 添加新设备 $\rightarrow$ 搜索匹配的蓝牙设备选中 $\rightarrow$ 下一步 $\rightarrow$ 输人蓝牙 
配对密码 一下一步, 已配对完成; COM 端口一新建发送端口， 选中所需机身端口 $\rightarrow$ 下一步 $\rightarrow$ 分配选择一个端口 $\rightarrow$ 完成 $\rightarrow$ 选 择配对设备 $\rightarrow$ 确定 $\rightarrow$ 连接 $\rightarrow$ 关闭 $\rightarrow$ 关闭 $\rightarrow$ 查看状态 $)$; 控制 点 $\rightarrow$ 管理 $\rightarrow$ 键入 $\rightarrow$ 坐标点库 $\rightarrow$ 增加 $\rightarrow$ 输入相应的 $X 、 Y 、 Z \rightarrow$ 属性选择“控制点” $\rightarrow$ 确定。

\section{4 点校正 (求参数) 方法}

手簿及软件正常工作状态时点校正操作，软件提供有两 种校准方式: (1)利用基站点校准: 利用变换前基站坐标和当前 基站的坐标进行校准; 2利用标记点校准:利用站前已经采集 坐标点和站后该点的坐标进行校准, 利用标记点校准流程。

\section{4 .1 同一第一次点校正 (两个已知点及以上)}

作业 $\rightarrow$ 计算 $\rightarrow$ 转换参数 $\rightarrow$ 设置当前已知坐标（将移动主 机整平对中至已知点上) $\rightarrow$ 增加相应坐标点 $\rightarrow$ 确定 $\rightarrow$ 设置 WGS84 坐标 $\rightarrow$ 获取当前 GPS 坐标 $\rightarrow$ 停止 $\rightarrow$ 确定 $\rightarrow$ 确定 $\rightarrow$ 确 定 $($ 以相同步骤增加其他点 $) \rightarrow$ 计算 $\rightarrow$ 查看参数 $($ 确定 $) \rightarrow$ 四参 数 (比例尺接近 1$) \rightarrow$ 确定 $\rightarrow$ 确定 $\rightarrow$ 应用 $($ 点校正 $)$ 。

3.4 .2 同一第二次点校正 (一个控制点)

作业 $\rightarrow$ 计算 $\rightarrow$ 校正参数 $\rightarrow$ 设置当前已知坐标 (将移动主 机整平对中安设至已知点上) $\rightarrow$ 直接输人当前坐标点 (或从点 库查找) 一确定; 设置 WGS84 坐标 $\rightarrow$ 获取当前 GPS 坐标 $\rightarrow$ 确

\section{（上接第 1 页）}

腐工作, 这对于岩土工程稳定性和安全性有重要的意义。抽水 试验的具体流程是: (1)做好抽水试验的准备工作。勘察人员可 以根据岩土工程基坑面积的大小,设置 2 3 个不同的抽水试 验对象, 方便数据对比。一般来说, 要最少设置 2 组, 2 组试验 均设置相同的抽水孔和检测孔，同时还可以在检测孔上布置 一根观测线, 保证可以对试验数据进行直观的分析 ${ }^{[1]}$ 。勘察人 员确保准备工作无误后就可以进行抽水试验。2抽水试验的 步骤是: 首先勘察人员在抽水试验开始前要对水位进行标注 和记录, 保证每组抽水试验的静水位起点一致, 也就是静水位 检测; 其次勘察人员要进行动水位检测和出水量计算, 在此操 作过程中, 勘察人员要将水位记录时间控制为每 30s/次, 直至 水位稳定或者变化不明显方可停止记录，为了保证水位测量 数据的准确性, 可以进行反复的抽水试验, 求出平均值; 对测 量到的数据信息进行系统的分析和计算,绘制图标, 将抽水试 验结果直观地展示出来, 为岩土工程施工方案的制订提供数 据支持。

\section{5 提升岩土工程基坑勘察水平的策略}

随着城市化进程的不断加快，岩土工程施工数量在不断
定 $\rightarrow$ 确定。

\section{5 放样测量}

点校正检核合格后,开始作业（软件操作可一台作业，也 可多台同时作业):工具 $\rightarrow$ 数据键入 $\rightarrow$ 点坐标库 $\rightarrow$ 导入, 自定 义格式 (点名, 坐标 $X$,坐标 $Y$,坐标 $H$ 与键入格式一致) $\rightarrow$ 导 入类型 $($ 放样点 $) \rightarrow$ 找到拷贝到手簿存储器文件 (格式与软件 支持相同 $) \rightarrow$ 确定 $\rightarrow$ 关闭 (返回) ; 点击测量 $\rightarrow$ 点放样 $\rightarrow$ 找到放 样点 $\rightarrow$ 选择 $\rightarrow$ 根据提示找到位置 $\rightarrow$ 测存 (提供点坐标, 并实测 检核)。点翻页键“乃”, 继续下一点的放样, 直至放样完成。

\section{4 结语}

在本项目测量中通过优化 GNSS RTK 操作方法及步骤, 精炼了基准站及移动站校正、放样测量及软件操作等操作程 序。更加体现了 GNSS RTK 在地面物探工程测量中的高精度、 高效率、机动灵活及操作简便快捷等特点。对本项目工程测量 质量和效益起到决定性作用，在后来类似项目中得到科技工 作者的认可,并推广应用。

\section{参考文献}

[1]GB/T 18314-2009 全球定位系统 (GPS )测量规范[S].

[2]高小六.GNSS RTK 技术在工程施工测量中的应用[J].民营科 技,2014(11):45.

增加, 对基坑勘察技术的要求也越来越高, 为了进一步提升岩 土工程施工质量和安全,勘察单位要结合基坑结构特征,选择 适宜的勘察技术进行基坑勘察。建筑企业要不断完善勘察体 制, 加强勘察人员的技术培训, 同时, 引进专业的技术人才, 提 升基坑勘察人员的专业技能。对于当前不规范的基坑勘察现 象, 国家有关部门要制定科学有效的市场监管体系, 确保岩土 工程基坑勘察数据真实准确。除此之外, 岩土工程基坑勘察从 业人员要始终坚持创新意识和创新精神，对现有的基坑勘察 技术进行创新和完善, 获取更多精准的勘察数据信息, 提升岩 土工程基坑勘察质量,减少基坑支护施工事故的发生。

\section{6 结语}

综上所述，目前岩土工程基坑勘察中所使用的勘察技术 主要有勘探孔、钻孔取样和原位测试及抽水试验三种, 基坑勘 察技术的应用对保证岩土工程施工质量有重要的意义。所以， 施工企业要给予足够的重视, 加大监控力度和创新力度, 保证 岩土工程的顺利开展。

\section{参考文献}

[1]向佐伟.某基坑工程岩土工程勘察技术探讨 [J].资源信息与工 程,2019,34(4):77-78. 